\title{
Post Mortem Contact by Fatal Injury Victims with Emergency Service Workers at the Scenes of Their Death
}

\author{
Richard E. Kelly, Ed.D. \\ Huntingtown, $M D$
}

\begin{abstract}
Ninety selected emergency service workers (68 police officers and 22 firefighter/emergency medical service personnel) were interviewed or completed questionnaires to determine if they had experienced a sense or feeling of "communication, presence, or attachment" from victims of fatal injury whom they had attended at death. Remarkably similar or related experiences were reported by 28 percent of the subjects. Reports ranged from simple affirmative responses to detailed descriptions. Partners corroborated two events, and several accounts described the location of the victim as above, over their shoulder, or looking down upon them, similar to accounts reported by survivors of near-death experiences. None of the subjects reported or displayed any symptoms of mental illness beyond usual posttraumatic event reactions, and all were experienced in their respective professions. I discuss the nature of these contacts and why similar reports of contact by deceased victims with attendees at their death have not appeared elsewhere; and I suggest studies with a more refined question protocol with other populations, such as hospital emergency room personnel, hospice care staff, chaplains, other clergy, and funeral home workers.
\end{abstract}

KEY WORDS: emergency service workers; traumatic injury; near-death experience.

Since all major religions, representing the beliefs of the vast majority of people in the world, allow for some form of continued or concurrent life separate from the physical, the possibility of discarnate interaction

Richard E. Kelly, Ed.D., a retired Detective Lieutenant in the Massachusetts State Police, served for 13 years with that agency's Psychological Services Unit. He served as Chief of the Health and Safety Branch for the United States Marshals Service for seven years, is still working in federal law enforcement, and is a licensed clinical professional counselor. Reprint requests should be addressed to Dr. Kelly at 1420 Shaw Drive, Huntingtown, MD 20639; e-mail: kellys3md@aol.com. 
in general is credible and indeed is documented throughout religious and secular media. Narrowing the focus to the moments surrounding death, particularly one that is unexpected, one can postulate that the dead or dying individual might be confused, vulnerable, or frightened during the transition from physical life. Stephen Levine (1982, p. 279) remarked on this very question:

I now see that a sudden death may not be as fortunate as we have been conditioned to believe... Someone who is popped out of their body without much preparation, like a teenager in the fullness of life who has a fatal accident, may after the smoke clears wonder, "What the hell was that all about? What happened to my body? I can't be dead because this is still happening around me."

Near-death literature abounds with reports of individuals observing the scenes of their near-death experience (NDE), often from a position of "hovering above," and many confirm the Levine hypothesis of disbelief and confusion (Greenhouse, 1974; Greyson and Flynn, 1984; Moody, 1975; Rawlings, 1978; Ring, 1980; Sabom, 1982). Levine (1982, p. 280) cited an account of a friend who reported watching police and fire personnel during an NDE in the aftermath of a car crash and trying to decide whether or not to get in the ambulance with his body.

Many NDE accounts describe attempts by the dying or dead individual to contact people at the scene, nearly unanimously without success. The inability to make contact was first noted in Raymond Moody's work with individuals describing near-death experiences:

To complicate the fact that he is apparently inaudible to people around him, the person in a spiritual body soon finds that he is invisible to others. The medical personnel or others congregating around his physical body may look straight towards where he is, in his spiritual body, without giving the slightest sign of ever seeing him. (Moody, 1975, p.44)

Moody's subjects further related that they were unable to grasp or touch, and he cited an example of an individual who attempted to resuscitate himself unsuccessfully $(1975$, p. 53). This physical world impotence may also explain the limited number of reports of attempts by NDErs to interact with individuals at the scene, in that their early awareness of ineffectiveness may preclude such attempts. This lack of contact by NDErs is not due to lack of clarity of the scene, because experiencers have often provided vivid accounts of people, places, and things surrounding their NDE. In several instances they described in minute detail the medical procedures, setting, and conversations taking place around them (Sabom, 1982). 
If we begin with the presumption that there exists some form of continued life after physical death, irrespective of duration or nomenclature, and we accept the concept that a traumatic death might engender more fear, panic, or confusion than a more expected or planned death, then we can look to the scenes of sudden deaths as a more likely venue for communication attempts by the dying or dead with those who are around them. However, a review of the literature in trauma, thanatology, and near-death studies found no references describing interaction between emergency service workers and the dead they had attended.

\section{Preliminary Study}

In a preliminary study of treatment following critical incidents by a major police department psychological services unit, six emergency service workers (four police officers and two paramedic firefighters) reported feelings of contact by deceased victims at the scenes of fatal injury in the form of sensing a presence, attachment, or plea for help (Kelly, 1992). These reports were collected over several years, and by the fourth account ultimately weighed against dismissal as anomaly and raised a question of frequency of this phenomenon.

The six reports described in my earlier study involved four separate events, two of which were described independently by partners. In all cases the emergency service workers were experienced in their work and had attended deaths previously. In all cases the emergency service workers were viewed to be psychologically intact as evidenced by years of physical and emotional health, absence of prior psychiatric history, and appropriate reactions to the traumatic events. In all cases the feelings described were reluctantly presented. Indeed, in both cases described by partners, there had been no discussion of their feelings of attachment, presence, or communication until counseling slowly and cautiously revealed them. In the two cases experienced by partners, a sense of the deceased was identified as hovering above them and watching, and in both cases the partners corroborated both the feelings and the perceived location.

In the first case, the emergency service workers felt the victim was "clinging" to them in some way that was difficult to describe. In the second case the emergency service workers felt the victim was watching them. In the third case the emergency service worker felt a bonding to the deceased immediately after eye contact, and later as if "he was on your back." In the fourth case eye contact was part of a continuing, 
personal, and strong connection to a victim along with a nagging sense of unfinished business.

In all cases the feelings lingered, and in all cases the feelings were personal. That is, the emergency service workers were clear that their perceptions were connected to the victims, rather than to the event in general. A planned, conscious, reverent act was a key element to the release of the feelings reported for each event. The traumatic death scene is not conducive to the subtle and liminal sensations reported by the emergency service workers described above. The fact that these feelings were identified by these emergency service workers may speak to the strength of the attempt of the deceased, the sensitivity of the receiver, or the lack of common reporting of such experiences by emergency service workers and other populations.

I present in this paper a larger study of 90 emergency service workers (68 police officers and 22 firefighter/emergency medical service personnel) involving questionnaires and interviews regarding similar or related experiences in their careers.

\section{Methods}

Subjects were 90 emergency service workers, including 67 police officers and 23 firefighter/emergency medical technicians serving rural and urban central Massachusetts. They were predominantly male ( 88 percent), had an average age of 39.7 years and an average occupational seniority of 15.9 years.

Subjects were drawn from three sources. During training sessions on critical incident stress, colleagues distributed 130 written questionnaires to trainees, after providing a brief description of the project and answering questions about the study design. Respondents had the option of identifying themselves or answering anonymously. Sixty-two subjects responded to the written questionnaire, for a 48 percent response rate. Of those 62 respondents, 12 (20 percent) chose to identify themselves.

In addition, 28 subjects, including the original six, were drawn from a review of 2,548 case files of a police counseling unit and interviewed. To mirror the profile of the original four cases and minimize other variables of experience or distortion, selections were made according to the following criteria: The subject had to be an emergency service worker with a previously established confidential relationship with the researcher, and with no indication of mental illness or extraordinary distress, who 
had been physically near the deceased; and the case had to involve a sudden or traumatic fatal event, and could not be the emergency service worker's first death experience.

As an additional control and data search, the five living original subjects were reinterviewed (the sixth had died of cancer).

Pertinent to this report, both questionnaire respondents and interviewees were asked the question: "Have you ever felt a 'presence,' 'communication' of some kind, or a feeling of 'attachment' from a deceased victim?"

\section{Results}

Of the 90 subjects, 30 (33 percent) answered positively, and 60 (66 percent) of the responses were negative, unable to be determined, or unanswered. Five of the positive responses, all from the anonymous questionnaire group, did not provide enough information to be sure that they met the criteria for acceptance. Further qualitative analysis of the 25 remaining positive responses showed 10 to be missing one or more of the criteria and were therefore classified as "related experiences." What remained were 15 respondents ( 17 percent) who reported having experienced feelings of presence, communication, or attachment by deceased victims. These 15 responses were strongly supported by the 10 others reporting "related experiences."

Viewing the data by number of occurrences, these 15 respondents described 23 specific events. Excluding the "related" cases, the 15 subjects fully meeting criteria accounted for the following: one subject described three experiences; three subjects reported feelings of presence at all death scenes; one subject identified 17 events; and two other respondents, due to their lengthy careers, estimated 100 experiences each. These responses combined equal 229 reports of presence.

The 15 subjects reporting presence that fully met the criteria showed no evidence of fabrication or mental illness. Those that addressed the issue were clear that the source of their feelings was not imagination or "internal voices." All offered their information freely and without coercion. They showed good psychological function before, during, and after the event; had prior experiences with death; noted the presence as unusual; were not extremely religious; and accepted a spiritual explanation of the presence as plausible.

Most of the dead were victims of traumatic death. Other experiences included contacts with deceased friends, coworkers, or relatives. The 
contacts were described not as apparitions, visions, or heard voices, but rather as thoughts, ideas, images, or feelings. Sensory input was very limited, but the conviction of the contact was strong. These emergency service workers, who were familiar with death and well grounded in the physical world, were quite aware of the unusual character of these experiences, but were equally clear that they were real.

An interesting disclosure came from one particular subject identified from the case file search as a result of involvement in a fatal shooting of an assailant by the subject and his partner. During the interview for this study he reported that he never felt a "communication," but "always has feeling at fatal scenes that they're still there... always above, just above, like over the shoulder, hovering." He went on to say it was as if they were watching him. The feeling for him is stronger on a call when he is alone, and it is always strong enough to cause him to act respectful, as if they can hear him. When asked if there were any occasions where he did not feel their presence he answered, "Yeah, the guy in [the shooting]--nothing with him."

As noted above, the five living original subjects were reinterviewed. My account of the earlier reports was presented for verification of accuracy and supplementation. All four accounts were accepted as highly accurate as recalled from subject recollections that were still vivid and unchanged in depiction.

\section{Discussion}

Emergency service workers are experiencing contacts with the spirits of deceased victims around the time of their deaths from traumatic injury. From this group of 90 veteran emergency service workers, 25 described contact with deceased individuals in the form of a presence, communication, or feeling of attachment. All study subjects were experienced and psychologically intact. Not surprisingly, none had discussed their experiences with others.

Fifteen of the 90 reports met study criteria, with an additional 10 describing related experiences of contacts or awareness with deceased individuals. Some of the accounts were bare, but several offered detailed and clear accounts of their interactions.

As a matter of perspective, the total number of deaths associated with the sample group should be viewed. Combined, the 90 respondents report experience with more than 7,000 deaths in their cumulative 1,431 years of emergency service worker experience. In that context, the 229 
reported episodes occurred in only 3 percent of the total deaths attended. However, it gains significance if seen that more than 200 reports of presence are generated from a group 90 subjects, and even more strikingly from a subset of only 15 from that group.

\section{Methodological Considerations}

The method used here was systematic and compliant with current research norms. The subjects were, perhaps, as far as one might imagine from being a group prone to superstition or hysteria. On the other hand, the study had some limitations.

Each of the data collection means had weaknesses. The original six cases, which arose in the context of counseling in a police department psychological services unit, could have been influenced by the counselor. The additional 22 subjects identified by subsequent case file search might also be subject to this same bias. Subject suggestibility by the researcher was protected against, in part, by the predetermination of questions and the selection of subjects by preset criteria.

There were several compromises made in the design of the questionnaire, including brevity, in order to increase response rates, and lack of precision of terminology, to allow for more open-ended reports. Allowing for anonymity also led to abbreviated and cryptic data that could not be clarified. One response in particular illustrates this frustration: the respondent answered the question of presence with, "Yes-I would explain, but it would take too much time. I can only say that I feel a strong presence at the scene of a death. I have always thought that there must be some way to communicate with 'it,' but I have not been able to understand this feeling completely."

The three sources of cases acted as a balance to one another. The original six subjects provided in-depth, client-directed information, collected prior to hypothesis formation. The case file follow-up took advantage of a previously established trusted relationship between researcher and interviewee, and allowed for the clarifications that interviews can best provide. The questionnaire offered a nonthreatening vehicle for disclosure. The three methods acted as an internal validation, and similar cases of presence were reported from all three sources.

As an initial investigation of the prevalence and substance of interaction between emergency service workers and the spirits of deceased victims of traumatic injury, this work is sufficient. It is not, however, overwhelming in its scope, sample size, and randomness of subject selection. Refinements are needed in data collection to elicit 
better descriptions of the experiences. Larger samples are needed with proper controls for random selection. Ideally, the scope of the inquiry should be extended to other groups with increased likelihood of similar contacts, such as hospital emergency room personnel, hospice care staff, chaplains, other clergy, and funeral home workers, with proper control group comparisons. Other studies examining variables related to who senses these feelings and under what conditions it occurs should follow a better understanding of prevalence.

\section{Theoretical Implications}

The implications of this study are significant. Twenty-eight percent of a sample of emergency service workers reporting these events is noteworthy. Although these cases may be similar in some ways to studied and anecdotal reports of near death experiences, they raise several points of interest. First, they focus attention on emergency service workers as a potentially important group in connection with the activities of the dying. They also suggest that attempts by NDErs and/or dying individuals to communicate with those around them may be more successful than previously thought. And they offer evidence from a highly credible group that such feelings are being sensed from the living, nonnear-death receiver.

The extension of NDE accounts as a model for seeing these events from the victim's view is helpful. However, caution is suggested in that, by definition, NDE reports are from those who have returned to provide them, and we cannot necessarily assume that the experience of the dead is the same as that of the dying who survive.

The evidence showing an interaction with these emergency service workers at the scenes of fatal traumatic injury naturally draws us to speculate as to nature and cause. The limits of the data are reached at supporting prevalence, and do not supply definitive insight as to what is occurring between victim and rescuer. The victim mindset of fear, panic, and confusion suggested by Levine (1982) is plausible. The logic that such a mental state would be more often presented to emergency service workers is equally plausible. Added to these assumptions is the potentially salient notion that emergency service workers are generally benevolent in their occupational motivation, irrespective of their possibly calloused protective external presentations. And lastly, these emergency service workers are sufficiently experienced at their craft to allow them to be less overwhelmed by the events at scenes that might overload the senses of others. It may be that, for those spirits wrapped 
in confusion, the emergency service worker is available, at least subliminally willing, and capable of sensing an attempt for communication from a "troubled soul."

\section{Clinical Implications}

This report does not address the impact, therapeutic response, and spiritual reaction by the emergency service workers to these events. They are in themselves significant. When emergency service workers and others are faced with contacts or feelings that are spiritual, then their best response may also be spiritual.

Keeping such information suppressed serves no purpose except to conform to common contemporary cultural norms to avoid discussion of all matters related to death and dying. To a lesser extent, these same societal restraints also prefer to relegate all things of spirit to superstition or hysteria.

One might ask, if contact by the deceased with emergency service workers is happening so often, why it has not been talked about before. The answer may be as simple as that no one has asked. There is a possible parallel with NDEs, where for centuries there were scattered references in religious and medical literature, but the more popularized and accepted notion of NDEs is more a product of the latter part of the 20 th century. Or perhaps, these awarenesses are part of a larger, more gradual recapturing of respect and understanding of things liminal, sensory, intuitive, or spiritual.

\section{References}

Greenhouse, H. B. (1974). The astral journey. New York, NY: Avon.

Greyson, B., and Flynn, C. P. (Eds.). (1984). The near-death experience: Problems, prospects, perspectives. Springfield, IL: Charles C Thomas.

Kelly, R. E. (1992). Present at the moment of death: Implications for counseling of emergency service personnel. The Forum Newsletter: Newsletter of the American Association of Death Education and Counseling, 17(4), 1 and 17-19.

Levine, S. (1982). Who dies? New York, NY: Doubleday.

Moody, R. A. (1975). Life after life. Covington, GA: Mockingbird Books.

Ring, K. (1980). Life at death: A scientific investigation of the near-death experience. New York, NY: Coward, McCann and Geoghegan.

Rawlings, M. (1978). Beyond death's door. Nashville, TN: Thomas Nelson.

Sabom, M. (1982). Recollections of death: A medical investigation. New York, NY: Harper and Row. 